\title{
Rreproduction of experimental spatio-temporal structures in traffic flows using mathematical model based on cellular automata theory
}

\author{
A. Chechina ${ }^{1}$, N. Churbanova ${ }^{1,2}$, M. Trapeznikova ${ }^{1,2}$ \\ ${ }^{1}$ Keldysh Institute of Applied Mathematics RAS, Moscow, Russia \\ ${ }^{2}$ Moscow Automobile And Road Construction State Technical University (MADI), Moscow, Russia
}

\section{Article Info}

Received Dec $31^{\text {th }}, 2018$

\section{Keyword:}

Cellular automata

Traffic flow modeling

\begin{abstract}
The paper deals with mathematical modelling of traffic flows on urban road networks using cellular automata theory. Two versions of the model based on Nagel-Schreckenberg traffic flow model were created. They both are multilane, include complex driver behaviour algorithms and allow simulating traffic on various road elements and on road networks. One of the models is using "slow-to-start" concept that represents the fact that it takes drivers more time to accelerate when they just start moving in comparison with the situation when their speed is already above zero.
\end{abstract}

The goal of the work was to reproduce experimental spatio-temporal patterns on traffic velocity diagrams with the created models and to research the difference between them and to determine the range of the applicability for each.

Parallel algorithms of realisation for the models are created. Computations are carried out for each element of the road ( $\mathrm{T}$ or X type intersection, on-ramp, road fragment with widening or bottleneck, straight road fragment, etc.) separately and in parallel, that allows performing calculations on various complex road networks. Different kinds of average characteristics (such as, for instance, the capacity of the crossroad) can be also obtained using the created program package.

Computations show that the results obtained are in an agreement with experimental data and therefore can be used for practical traffic flow modelling in cities.

\section{Corresponding Author:}

Antonina Chechina,

Keldysh Institute of Applied Mathematics RAS,

4 Miusskaya Square, Moscow, Russia.

Email: chechina.antonina@yandex.ru

\section{Introduction}

The cellular automata theory (CA), first proposed and developed by John von Neumann in the mid-twentieth century, has found its application in many fields of science. With its help, economic, social, technical, biological and other processes are modelled.

Since 1992, when Kai Nagel and Michael Schreckenberg [1] proposed to apply the theory of cellular automata to transport modelling, scientists from around the world have created many variants of traffic flow models based on it. Previously, this approach seemed to be the most promising for a detailed description of local road situations at short distances, since the models are quite flexible due to the ability to implement any driver strategy without significant algorithmic costs. However, in connection with the capabilities of the currently 
existing ultra-high-performance computing equipment, models of this type can also be successfully used to simulate traffic on large road networks.

Due to the development of computer technology, it became possible to take into account more and more nuances in the behavior of traffic participants in the models created, and due to the development of the transport flow theory itself, new ideas about the patterns observed in the measurement data appeared. Also in recent times, a large amount of data from sensors and surveillance cameras has been collected and stored, which means that the requirements for the mathematical models being created are increasing.

\section{The developed models}

\subsection{The original model}

The original cellular automata model created by authors presents a generalization of the classic NagelSchreckenberg model [1] to a multilane case with various driver behaviour algorithms included (see [2], [3], [4] for details).

The road is divided into equal cells. As is usual for traffic CA models, a cell is 7.5 meters long and one lane wide, the time step is 1 second. The cell can be either empty or occupied by a single vehicle. Each car has a set of parameters: unique ID, maximum speed, current speed and final destination; its driver can be 'cautious' or 'aggressive', 'cooperative' or not ([2]).

\subsection{The 'slow-to-start' model}

According to the classic one-lane Nagel-Schreckenberg rules, the driver checks if the next cell is empty, and if it is, he starts moving. But there is another class of models - 'slow-to-start' - where vehicles begin their movement only on condition that there is more than one free cell in front of them [5]. This rule was included in the model so that cars did not disperse too quickly from the place of the traffic jam. It allows to reproduce the effect of hysteresis that is observed during the transition from the free flow phase to the synchronized flow phase, depending on random processes in the traffic flow.

According to the three phase theory by B. Kerner [6], there are three phases in the traffic flow: $\mathrm{F}$ is the phase of free flow, $S$ is the phase of synchronized flow and $J$ is the phase of wide moving jams. Due to the instability of the flow, for example, due to the effect of over-acceleration [7], phase transitions can occur spontaneously. As experimental data shows, models of the 'slow-to-start' class reproduce such phase transitions more successfully.

To include the 'slow-to-start' rule, the set of appropriate conditions was added to the created model.

\section{Tests}

Several numerical experiments were carried out in order to verify the proposed model. A problem of velocity field patterns for a road fragment with an on-ramp was analysed. On the intersection of two roads, one of which is a main road with priority pass, and the other one is a secondary road, where drivers have to wait for a large enough gap in the flow on the main one to enter (Fig. 1). Q1 is the influx flow on the main road, Q2 is the influx flow on an on-ramp.

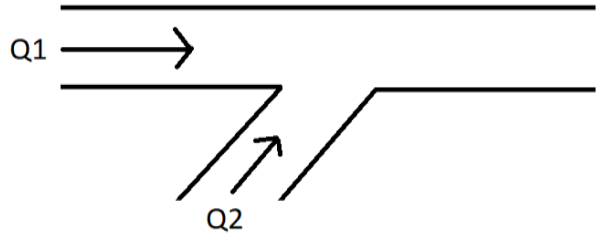

Figure 1. The road with on-ramp. Q1 is the flow on the main road; Q2 is the flow on the secondary road.

The experimental data for this test problem, taken from [6], are presented on Figure 2. At time moment $\mathrm{T} \sim 10 \mathrm{~min}$ the spontaneous F-S phase transition occurs. 


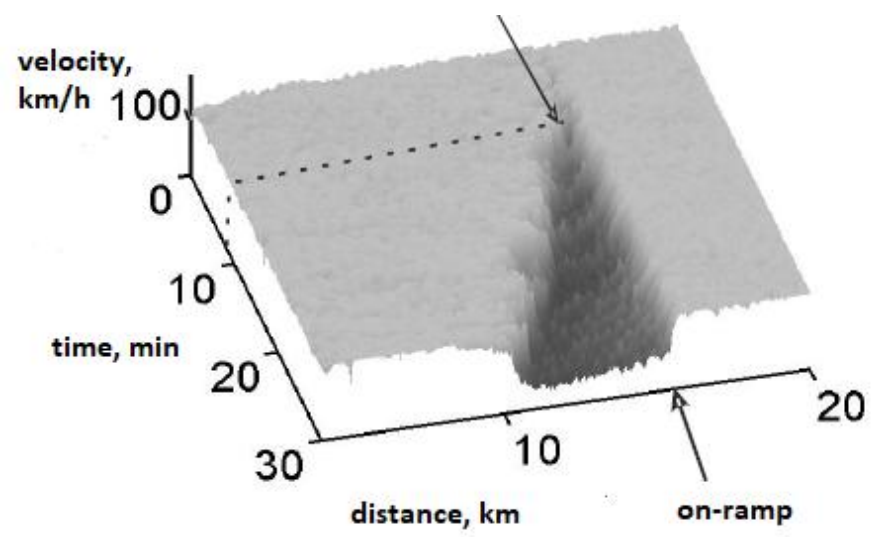

Figure 2. The velocity field on a road with an on-ramp bottleneck - experimental data [6].

The same situation was simulated using both models. The results obtained with both models are shown on Figure 3. Ox axis (T) is time in minutes, Oy axis $(\mathrm{X})$ - distance in kilometers, vehicular speed $\mathrm{V}$ is represented with color, from red $(\mathrm{V} \sim 0 \mathrm{~km} / \mathrm{h})$ to dark blue $(\mathrm{V} \sim 100 \mathrm{~km} / \mathrm{h})$. The on-ramp entrance is situated at the point $\mathrm{X}=3.5 \mathrm{~km}$.

As can be seen from the figures, both models adequately reproduce the F-S phase transition: small fluctuations in speed at the initial moment of time gradually become more substantial, a drop in speed occurs and the flow becomes synchronized.
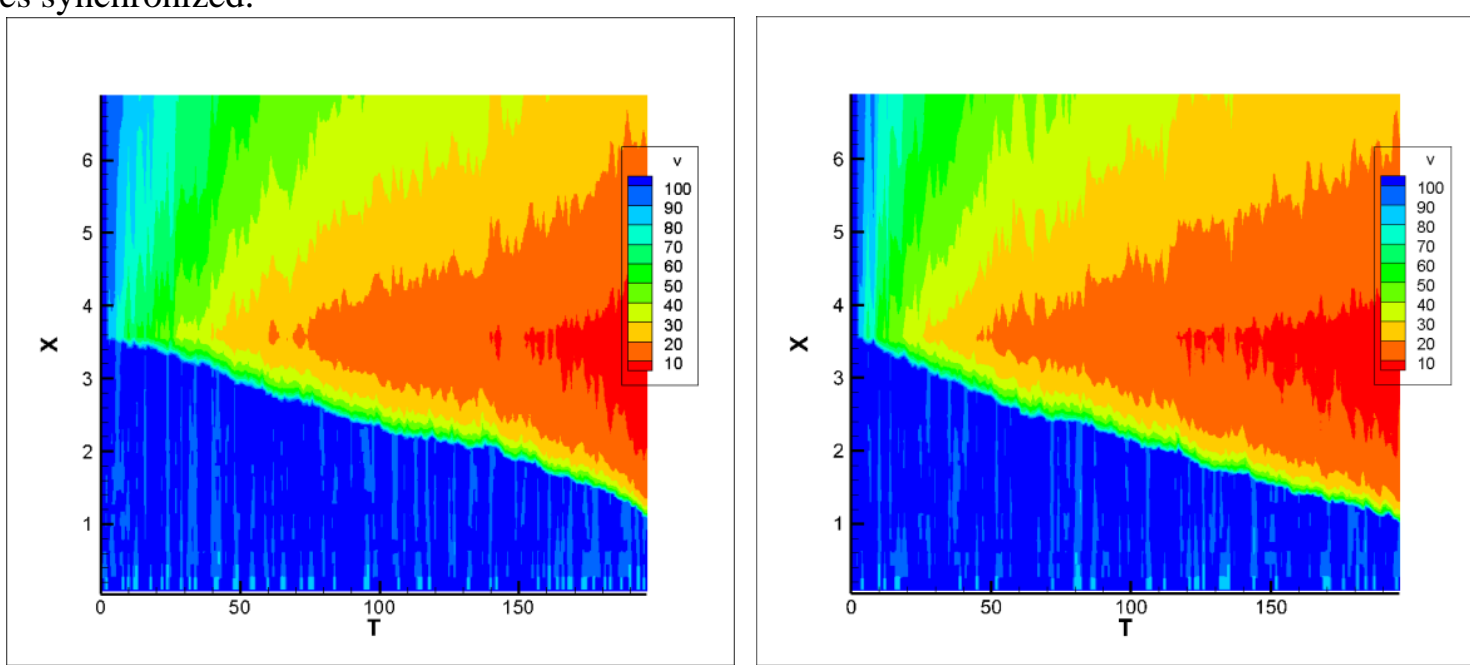

Figure 3. The velocity field on a road with an on-ramp bottleneck - numerical experiment results. Original model (on the left), slow-to-start model (on the right). Q1=20 veh/min; Q2=10 veh/min.

Traffic structures in real data, obtained in different parts of the world, are always similar and look like stripes of lower velocity (red) on a spatio-temporal diagram (see Figure 4). Ox is time in hours and minutes, Oy distance in kilometres, colour corresponds to vehicles' speed. Red regions have low speed (traffic jam), yellow regions - higher speed (congested flow), white regions - maximal speed (free flow). 


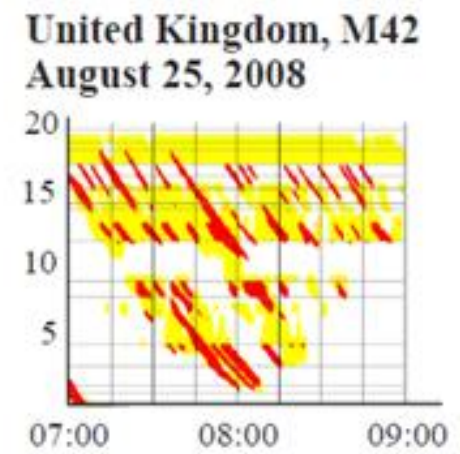

\section{Germany, A40-East June 1, 2007}

USA, US-I405-South

March 4, 2003

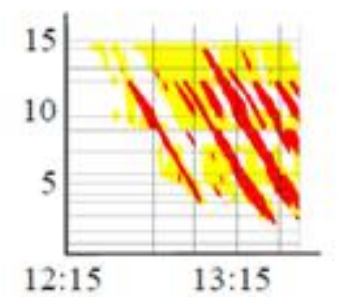

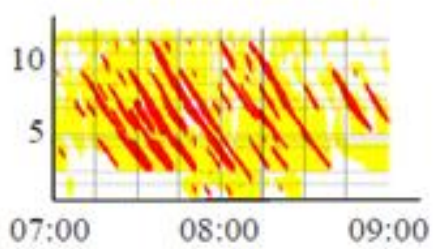

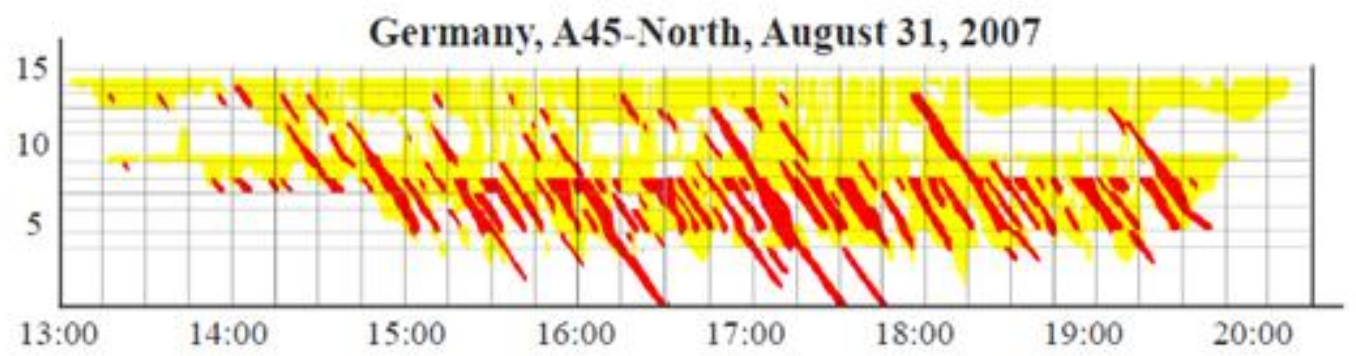

United Kingdom, M42, January 11, 2008

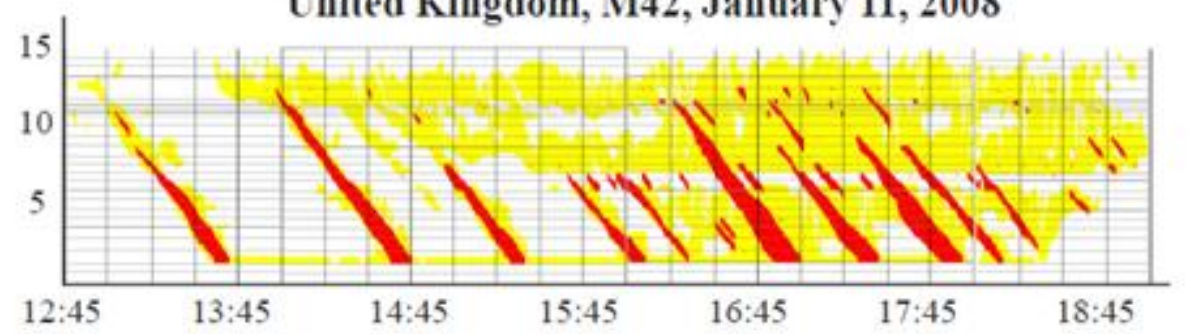

Figure 4. Experimental spatio-temporal patterns in traffic flows (from [6]).

In order to compare test predictions with these data and see if our models would reproduce said patterns, we once again simulate traffic on a road with an on-ramp bottleneck (Figure 1). This time, Q1 stays constant throughout the calculation, but Q2 drops down to zero at the time moment $\mathrm{T}=100 \mathrm{~min}$.

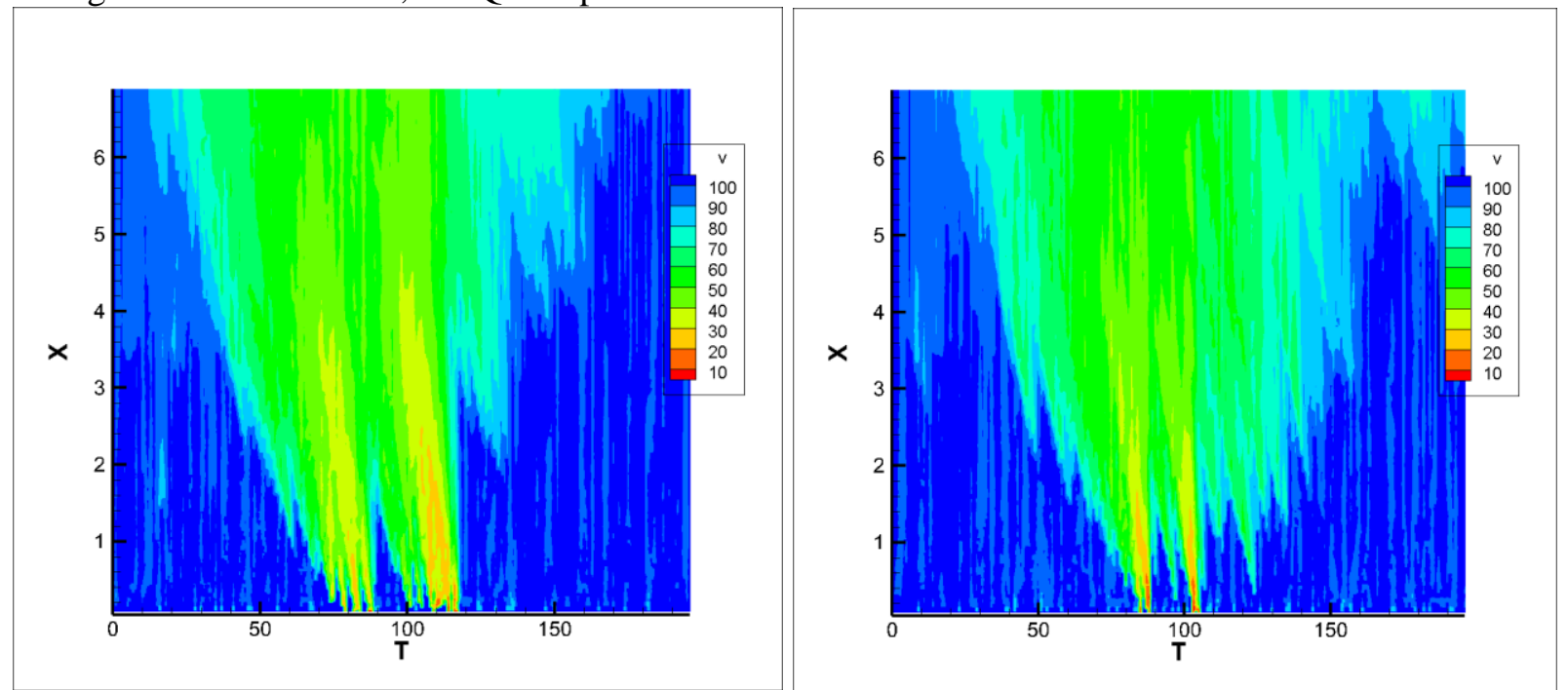

Figure 5. Spatio-temporal structures in the traffic flow near on-ramp. Original model (on the left), slow-tostart model (on the right). Q1=21 veh/min; Q2=2 veh/min if $\mathrm{T}<=100 \mathrm{~min}, \mathrm{Q} 2=0$ if $\mathrm{T}>100 \mathrm{~min}$. 

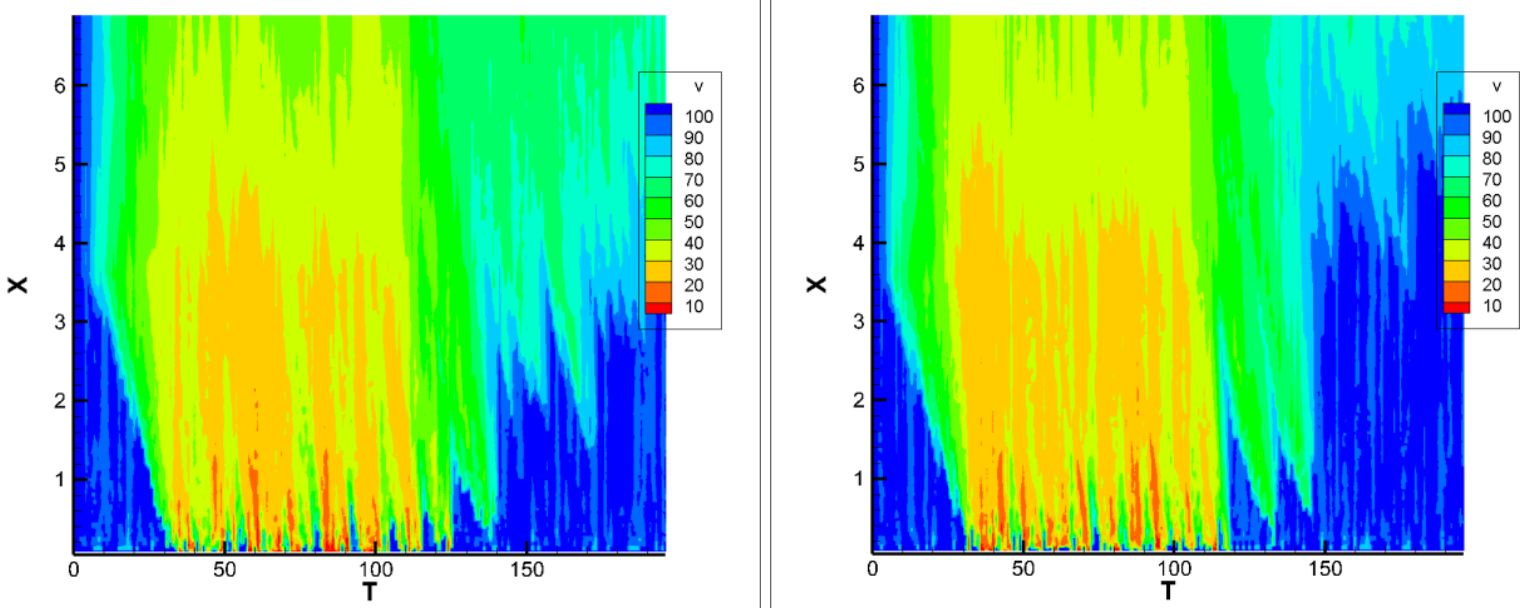

Figure 6. Spatio-temporal structures in the traffic flow near on-ramp. Original model (on the left), slow-tostart model (on the right). Q1=22 veh/min; Q2=6 veh/min if $\mathrm{T}<=100 \mathrm{~min}, \mathrm{Q} 2=0$ if $\mathrm{T}>100 \mathrm{~min}$.
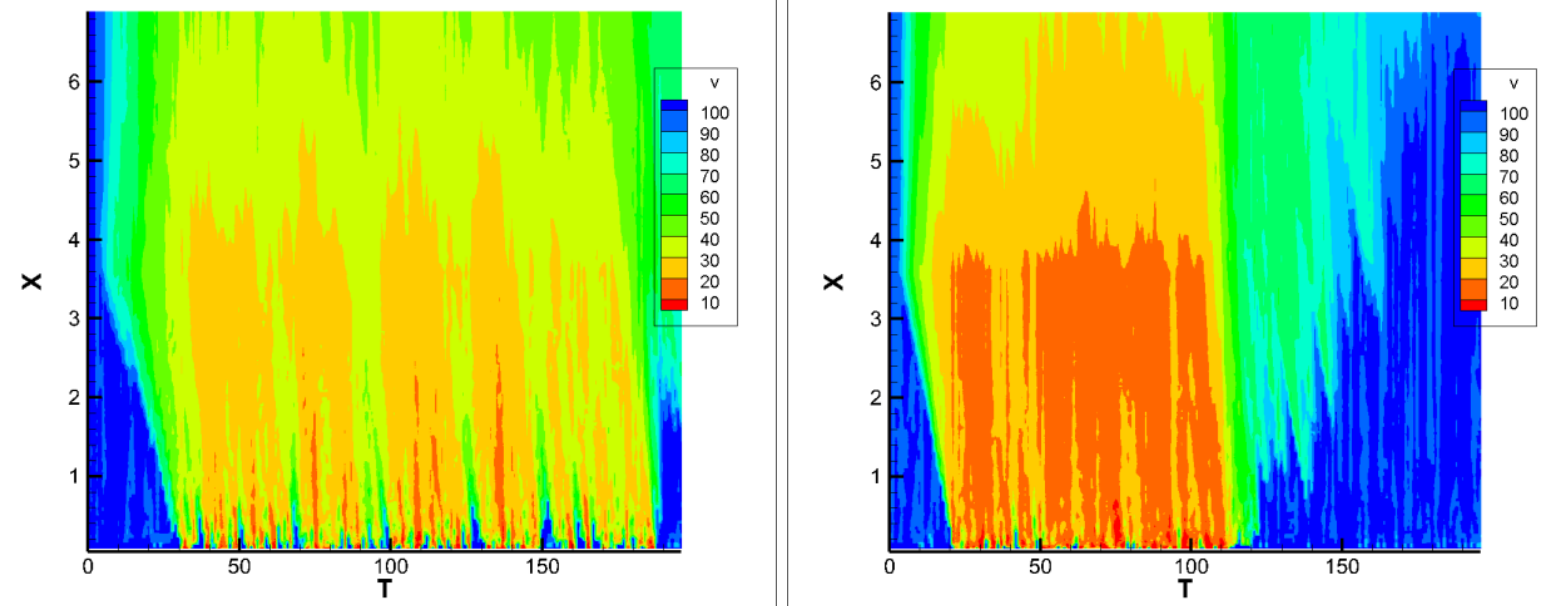

Figure 7. Spatio-temporal structures in the traffic flow near on-ramp. Original model (on the left), slow-tostart model (on the right). Q1=25 veh/min; Q2=9veh/min if T<=100 $\mathrm{min}, \mathrm{Q} 2=0$ if $\mathrm{T}>100 \mathrm{~min}$.

The vehicle velocity on different points of the road at different time moments was calculated. The results were averaged out for easier comparison.

On Figures 5, 6, 7 the results of computations carried out using the original model and the slow-to-start model for different values of Q1, Q2 are presented. $\mathrm{X}$ is distance in kilometres, $\mathrm{T}$ is time in minutes. The on-ramp entrance is situated at the point $X=3.5 \mathrm{~km}$. The color corresponds to vehicles' speed. Ox axis (T) is time in minutes, Oy axis $(\mathrm{X})$ - distance in kilometers, vehicular speed $\mathrm{V}$ is represented with color, from red (V 0 $\mathrm{km} / \mathrm{h}$, traffic jam) to dark blue (V 100 km/h, free flow).

As is seen on the figures, spatio-temporal diagrams obtained in computations by both models have similar stripy structures as in experimental diagrams. It's also notable that, as in experiment, there are no direct F-J phase transitions - first free flow transforms to congested (F-S), and wide moving jams appear only in congested flow (S-J).

Both models in this test show similar results in computations, although the slow-to-start model naturally produces vaster regions of lower velocity on spatio-temporal diagrams. Therefore, it was interesting to find out in which cases the results obtained using the models would differ from each other more. 

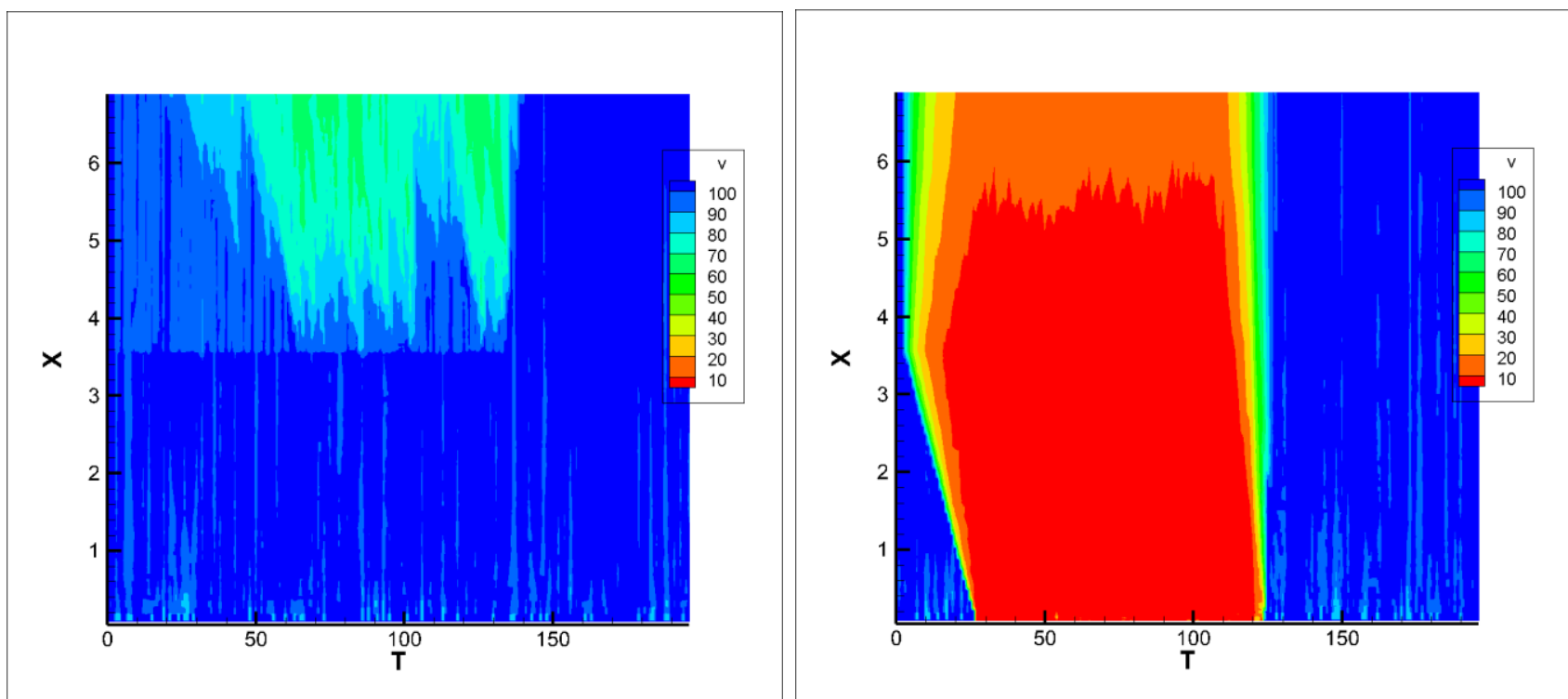

Figure 8. Spatio-temporal structures in the traffic flow near on-ramp, original model (on the left), slow-to-start model (on the right). Q1=20 veh/min; Q2=25 veh/min if $\mathrm{T}<=100 \mathrm{~min}, \mathrm{Q} 2=0$ if $\mathrm{T}>100 \mathrm{~min}$.

On Figure 8 spatio-temporal structures obtained in a similar simulation, but with more significant influx from the secondary road, are presented. In this case, the slow start results in a major congestion near the entrance that quickly extends both upstream and downstream.

Driver behavior, road and weather conditions and other features of vehicular traffic can vary significantly, therefore, for traffic flow simulation on the specific city road network, it makes sense to choose the variant of the model that suits the current situation best when calibrating the model.

\section{Conclusion}

The models presented in this work reproduce experimental spatio-temporal structures in traffic flows fairly well. Calculations using both models lead to similar results, with the only difference that the slow start leads to a decrease in speed in general.

In the future, it is planned to conduct a series of computational experiments to investigate how well they reproduce all features of Kerner's three phase theory. Also, both models will be tested on other test problems to further clarify the advantages and disadvantages of the 'slow-to-start' option.

\section{References}

[1] Nagel K., Schreckenberg M. A "Cellular automaton model for freeway traffic" J. Phys. I France, V. 2, P. 2221-2229, 1992.

[2] Chechina A., Churbanova N., Trapeznikova M., Ermakov A., German M. "Traffic flow modelling on road networks using cellular automata theory" International Journal of Engineering \& Technology (IJET), ISSN 2227-524X, Vol. 7, No 2.28, pp. 225-227, 2018.

[3] Antonina Chechina, Natalia Churbanova and Marina Trapeznikova. "Multilane Traffic Flow Modeling Using Cellular Automata Theory", EPJ Web of Conferences, V. 173, 06003, 2018.

[4] Bugaev A.S., Buslaev A.P., Kozlov V.V., Yashina M.V. "Distributed Problems of Monitoring and Modern Approaches to Traffic Modeling" 14th International IEEE Conference on Intelligent Transportation Systems (ITSC 2011), Washington, USA, pp. 477-481, 2011.

[5] Maerivoet S., Moor B. D. "Cellular automata models of road traffic" Physics Reports. Vol. 419, no. 1. Pp. 1-64, 2005.

[6] Kerner B. "The physics of Traffic" Berlin: Springer, 2004. 682 p.

[7] Kerner B., Klenov S., Hermanns G., Schreckenberg M. "Effect of driver overacceleration on traffic breakdown in three-phase cellular automaton traffic flow models", Physica A.: Statistical Mechanics and its Applications 392: 4083-4105, 2013. 